\title{
Combining Planning and Motion Planning
}

\author{
Jaesik Choi and Eyal Amir \\ Department of Computer Science \\ University of Illinois at Urbana Champaign \\ Urbana, IL, 61801 USA \\ \{jaesi,eyal\}@illinois.edu
}

\begin{abstract}
Robotic manipulation is important for real, physical world applications. General Purpose manipulation with a robot (eg. delivering dishes, opening doors with a key, etc.) is demanding. It is hard because (1) objects are constrained in position and orientation, (2) many non-spatial constraints interact (or interfere) with each other, and (3) robots may have multidegree of freedoms (DOF). In this paper we solve the problem of general purpose robotic manipulation using a novel combination of planning and motion planning. Our approach integrates motions of a robot with other (non-physical or external-to-robot) actions to achieve a goal while manipulating objects. It differs from previous, hierarchical approaches in that (a) it considers kinematic constraints in configuration space (C-space) together with constraints over object manipulations; (b) it automatically generates high-level (logical) actions from a $\mathrm{C}$-space based motion planning algorithm; and (c) it decomposes a planning problem into small segments, thus reducing the complexity of planning.
\end{abstract}

\section{Introduction}

Algorithms for general purpose manipulations of daily-life objects are still demanding (e.g. keys of doors, dishes in a dish washer and buttons in elevators). However, the complexity of such planning algorithm is exponentially proportional to the dimension of the space (the degree-of-freedom (DOF) of the robot and the number of objects) (Canny 1987). It was shown that planning with movable objects is P-SPACE hard (Chen and Hwang 1991; Dacre-Wright, Laumond, and Alami 1992; Stilman and Kuffner 2005). Nonetheless, previous works examined such planning in depth (Likhachev, Gordon, and Thrun 2003; Kuffner and LaValle 2000; Kavraki et al. 1996; Brock and Khatib 2000; Alami et al. 1998; Stilman and Kuffner 2005) because of the importance of manipulating objects. The theoretical analysis gave rise to some practical applications (Alami et al. 1998; Cortés 2003; Stilman and Kuffner 2005; Conner et al. 2007), but general purpose manipulation remains out of reach for real-world-scale applications.

Motion planning algorithms have difficulty to represent non-kinematic constraints despite of its strength in planning with kinematic constraints. Suppose that we want to let a

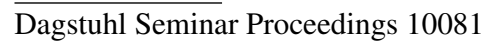

Cognitive Robotics robot push a button to turn a light on. CSpace ${ }^{1}$ can represent such constraints. However, the CSpace representation could be (1) redundant and (2) computationally inefficient because CSpace is not appropriate for compact representations. It could be redundant, because it always considers the configurations of all objects beside our interests (i.e. a button and a light). Moreover, mapping such constraints into CSpace would be computationally inefficient, because mapping a constraint among $n$ objects could take $O\left(2^{n}\right)$ evaluations in worst case. Thus, most of motion planning algorithms assume that such mappings in CSpace are encoded.

AI planning algorithms and description languages (e.g. PDDL (McDermott 1998)) have difficulty to execute realworld robots despite of its strength in planing with logical constraints. Suppose that we have a PDDL action for "push the button' which makes a button pushed and a light turned on. However, the PDDL description could be (1) ambiguous and (2) incomplete (require details). Given a robot with $m$ joints, it is ambiguous how to execute the robot to push the button, because such execution is not given in the description. Instead, it assumes that there is a predefined action which makes some conditions (e.g. a button pushed) satisfied whenever precondition is hold and the action is done.

Both methods solve this problem in different ways. Motion planning algorithms use abstractions to solve this problem. AI plannings use manual encodings. Although abstraction provides solutions in a reasonable amount of time in many applications, abstraction lose completeness. Thus, it has no computational benefit in worst cases. Although AI plannings have no need to search the huge CSpace, it requires manual encodings which are not only error-prone but also computationally inefficient.

We minimize manual encodings using the reachability of objects. That is, logical actions are extracted from a tree (planned by a motion planning algorithm), if the actions change the reachability of objects (i.e. a switch can be reachable by opening a door).

Our algorithm provides a path of a robot given following inputs: configurations of a robot and objects; constraints between objects; an initial state; and a goal condition. ${ }^{2}$ We use logical expressions to represent both spatial constraints

\footnotetext{
${ }^{1} \mathrm{CSpace}$ is the set of all possible configurations

${ }^{2}$ For each object, we provide a function which maps from a configuration to discrete states (labels) of objects, if discrete states
} 
in C-space (e.g. collision) and constraints in state space (we define them formally in section 4 . We automatically build a set of actions from a motion planner, while it was done by hands in previous works.

In detail, our algorithm unifies a general purpose (logical) planner and a motion planner in one algorithm. Our algorithm is composed of three subroutines: (1) extracting logical actions from a motion planner, (2) finding an abstract plan from the logical domain, and (3) decoding it into C-space. It extracts PDDL actions (McDermott 1998) from a tree constructed by a motion planner in $\mathrm{C}$-space. Then, it combines extracted actions with a given $K B_{\text {object }}$ (Knowledge Base) that has propositions, axioms (propositional formulas) and abstract PDDL actions. To find an abstract plan efficiently, we automatically partitioned the domain by a graph decomposition algorithm before planning. In the planning step, an abstract plan is found by a factored planning algorithms (Amir and Engelhardt 2003; Brafman and Domshlak 2006) which are designed for the decomposed domain. In decoding, a motion plan is found from the abstract plan.

We argue that the complexity of a planning problem is bounded by the treewidth of the encoded KB. One may think some analogy between the treewidth of $\mathrm{KB}$ in this paper and the number of mutually-interfering objects in the motion planning literature. However, the treewidth is more general expression because KB has more expressive power than the conventional $\mathrm{C}$-space. In addition, this work proposes two improvements in terms of efficiency. One improvement is to use a factored planning algorithm for the decomposed domain. The other is to encode actions on behalf of workspace which is much smaller than C-space.

This approach is a unique decomposition-based path planning algorithm. We minimize manual encodings which are required to manipulate objects. Both (kinematic) constraints of the robot, and constraints of manipulating object are considered in our planning. It is efficient because its efficiency depends only on the workspace (2D or 3D), when appropriate conditions are met. Moreover, our method calculates actions of a robot once and can reuse them for other tasks.

Section 2presents related works. Section 3provides a motivational example. Section 4explains our encoding to build a KB. Sections 5and 6show our algorithm. Finally, section 7. provides experimental results followed by the conclusion in section 8 .

\section{Related Works}

Here, we review the related works in two aspects: (1) using logical representation in robot planning; and (2) modifying the motion planning algorithm to achieve complex task (eg. manipulating objects). One may see the former way as topdown and the latter way as bottom-up.

(Alami et al. 1998) presents a well-integrated robot architecture which controls multiple robots. It uses logical representations in higher level planners and C-space based motion planners in lower-level planning. However, the combination of two planners is rather naive (manual).

are required for the provided constraints of objects $\left(K B_{\text {object }}\right)$.

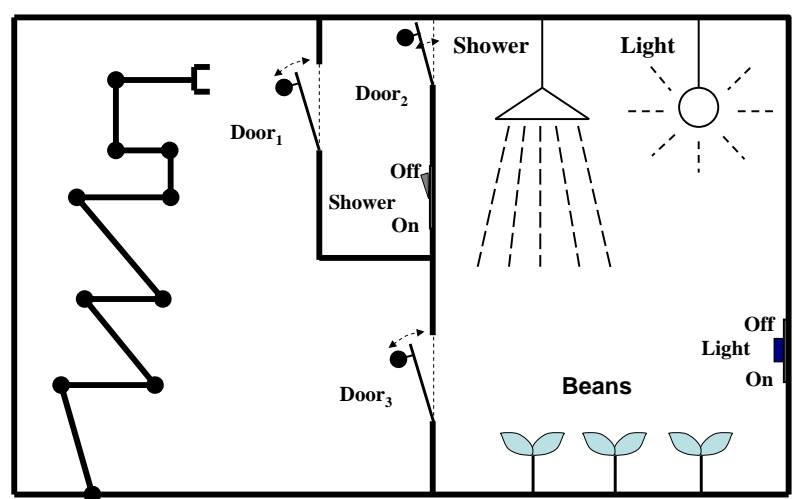

Figure 1: This figure shows an example of manipulating objects with a robotic arm. The goal is to take care of beans in a glasshouse. Beans require water and light everyday. The robot will provide water and light for beans. To accomplish this goal, the arm needs to manipulate objects such as doors and switches.

Recently, (Conner et al. 2007) provides an improved way to combine the Linear Temporal Logic (LTL) to control continuously moving cars in the simulated environment. ${ }^{3}$ However, their model is a nondeterministic automata, while our model is deterministic. Due to the intractability of nondeterministic model, their representation is restricted to a subset of LTL to achieve a tractable (polynomial time) algorithm. Experiments are focused on controlling cars instead of manipulating objects.

Motion planning research has a long-term goal of building a motion planning algorithm that finds plans for complex tasks (eg. manipulating objects). (Stilman and Kuffner 2005) suggests such a planning algorithm based on a heuristic planner (Chen and Hwang 1991) which efficiently relocates obstacles to reach a goal location. Recently, it was extended to embed constraints over object into the $C$-space (Stilman 2007). In fact, the probabilistic roadmap method (Kuffner and LaValle 2000) of the algorithm is highly effective in manipulating objects in detail. However, we argue that our algorithm (factored planning) is more appropriate in terms of generality and efficiency than a search-based (with backtracks) heuristic planner.

Other works also present efforts in this direction to build a motion planning algorithm for complex tasks. (Plaku, Kavraki, and Vardi 2008) solves a motion planning problem focused on safety with logical constraints represented with LTL . (M. Pardowitz 2007) focuses on learning actions for manipulating objects based on the explanation based learning (Dejong and Mooney 1986). They use a classical hierarchical planner in planning. (J. Van den Berg 2007) provides an idea that extracts the propositional symbols from a motion planner. The symbols are used to check the satisfiability of the planning problems. (S. Hart 2007) uses a potential field method to achieve complex tasks with two arms. However, the main interests of these works are not planning algorithm, or are limited to the rather simpler tasks.

${ }^{3}$ Any First Order Logic (FOL) sentences can be reduced to Linear Temporal Logic (LTL). Thus, LPL is a superset of FOL. 


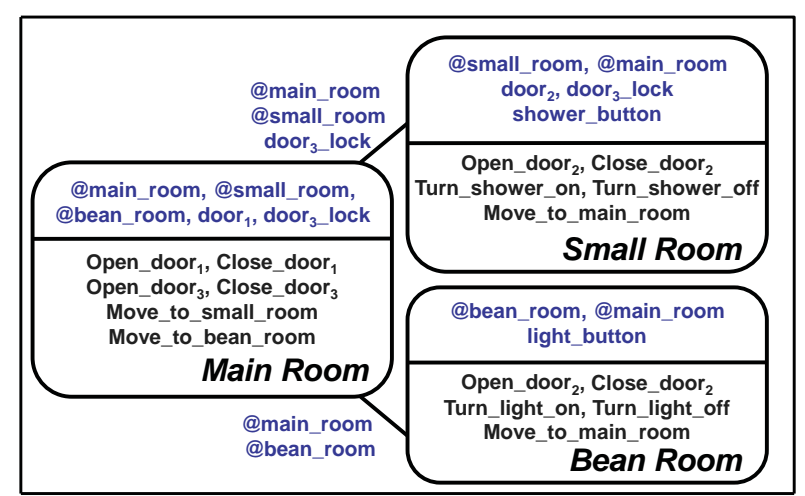

Figure 2: This is a possible tree decomposition for the toy problem of figure 1 . The shared propositions appear on edges between subgroups. For example, a proposition ('@door 3 _lock') is shared by two subgroups ('Main Room' and 'Small Room') because the proposition is used by actions of two subgroups (respectively 'Open(Close)_door 3 ' and 'Turn_shower_on(off)'). The KB is decomposed into small groups based on the geometric information (eg. the configurations of the room).

\section{A Motivating Example}

Figure 1 shows a planning problem. The goal is to provide water and light to beans. The robotic arm should be able to manipulate buttons in the spatial space to provide water and light. There are also non-spatial constraints. At any time either the shower is off or door 3 is closed or both.

The planner requires both a general purpose (logical) planner and a motion planner. It requires general purpose planner because the arm needs to revisit some points of C-space several times in a possible solution. The way points may include 'Open_door ${ }_{1}$ ', 'Close_door ${ }_{1}$ ', and 'Turn_light_on'. The state space can be different, whenever the robot revisits the same point in the C-space. It is certainly motion planning problem because the kinematic constraints of the arm should be considered. For example, the arm should not collide with obstacles, although the hand of the arm may contact objects.

Hierarchical planners have been classical solutions for these problems. A hierarchical planner takes in charge of high level planning. A motion planner takes in charge of low level planning. However, researchers (or engineers) need to define actions of the robot in addition to axioms among propositions for objects. Without the manual encodings, the hierarchical planner may need to play with the large number of propositions $\left(O\left(\exp \left(D O F_{\text {robot }}\right)\right)=\mid\right.$ discretized $C$-Space $\left.\mid\right)$ , when $D O F_{\text {robot }}$ is the DOF of the robot. With such naive encoding, computational complexity of planning become $(O(\exp (\exp (D O F s))))$.

Moreover, naive hierarchical planners often have difficulty to find solutions for the following reason. Firstly, it requires interactions between subgoals. For example, the arm must go into the "Bean room" and turn the "light" on (subgoal) before it goes into the "small room" and turn the "shower" on (subgoal). This is essentially the 'Susman anomaly' which means that the planner dose one thing (being in the Bean room) and then it has to retract it in order to

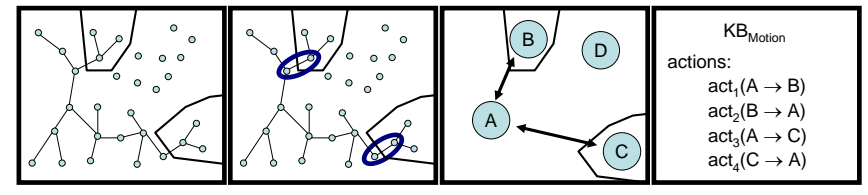

Figure 3: This figure illustrates a process to encode a motion plan into $K B_{M}$. The process is follows: (1) a motion plan (a tree) is built by a motion planning algorithm; (2) actions which changes the states of objects are found; (3) propositions are generated (and grouped) based on the found actions; and (4) a $K B_{M}$ is created. Here, we assume that we have a function which provides discrete states of objects given the configuration of an object in finding actions (2). In this figure, the door 1 in figure 1 and 2 is closed in a set of states $(A)$. The $d_{0 o r_{1}}$ is moved little in $B$. However, the $d o_{1} r_{1}$ is not fully opened. Thus, configurations in the area $D$ is not connected. The area $C$ corresponds to the pushed light button on figure 1 and 2.

achieve other goal (turning the shower on). Thus, it may require several backtracking in planning. Secondly, there are two ways of (in principle) achieving "on(light)": (1) going through the small room; and (2) opening door to the Bean room from the Arm-base room. Unless manual encoding is given by an engineer, The latter way (going through the small room) is fine from the perspective of hierarchical planning. However, it will not work in practice because the arm is not long enough (kinematics). Formally, there is no downward solution.

Thus, this toy problem shows that (1) hierarchical planning does not work with a naive (simple) encoding, and (2) a complete encoding is too complex to engineer directly. We are interested in general principles that underlie a solution to this problem.

In motion planning literature, hybrid planners are used to address these problem (Alami, Siméon, and Laumond 1989; Alami, Laumond, and Siméon 1997; Alami et al. 1998; Conner et al. 2007; Plaku, Kavraki, and Vardi 2008). However, these are either hard to engineer due to manual encodings, or infeasible to conduct complex tasks due to the curse of dimensionality of expanded C-space. The size of C-space of a hybrid planner exponentially increases with additional movable objects and given propositions. Thus, solving a complex problem may require extensive search.

Here, we seamlessly combine the general purpose planning and the motion planning. Our planner finds all reachable locations and possible actions that change states of object, states of propositions, or the reachable set of objects. ${ }^{4}$ Thus, high-level planner can start to plan based on the actions extracted by a motion planner. ${ }^{5}$ The number of actions and states can be different according to constraints of the robot.

However, the number of actions and states can be still intractable. To solve this problem, we partition the domain into the smaller groups of actions and states. For

\footnotetext{
${ }^{4}$ Here, we assume that we know states of objects without uncertainty as in (Conner et al. 2007).

${ }^{5}$ Our planner may have more actions and states than the handencoded case.
} 


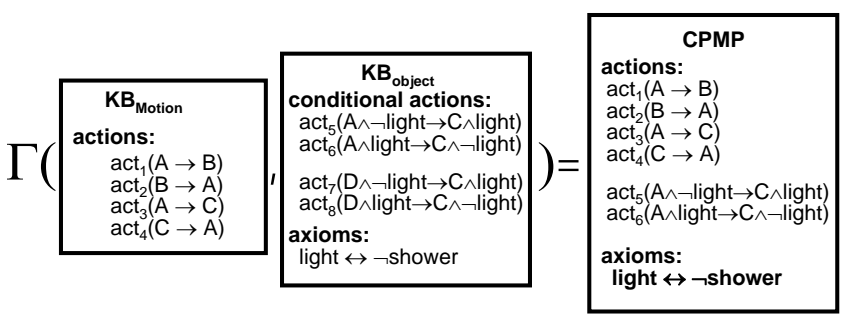

Figure 4: This shows an operation (or algorithm) to combine the extracted $K B_{M}$ with pre-existing $K B_{O} . K B_{O}$ is independently given in a general form to a robot. Thus, $K B_{O}$ can be reusable for robots with different configurations space. Meanwhile, $K B_{M P}$ is specific to a robot. Thus, some actions (e.g. $a c t_{7}$ and $a c t_{8}$ ) in $K B_{O}$ are invalidated by the $K B_{M}$.

example, the domain can be partitioned as shown in figure 2. It is composed of three parts: (1) operating the shower switch; (2) operating the light switch; and (3) operating in between. The partition can be automatically done with approximate tight bound (Becker and Geiger 1996; Amir 2001).

A factored planner(Amir and Engelhardt 2003) efficiently finds a plan with the partitioned domain. The partitioned groups are connected as a tree shape. In the factored domain, our factored planner finds all the possible effects of the set of actions in each factored domain. Then, the planner passes the planned results into the parents of the domain in the tree. In the root node, all the valid actions and effects are gathered. The planner finds a plan for the task, if it exists.

Then, we use a local planner to find a concrete path in Cspace at the final step. However, there is no manual (explicit) encoding (eg. 'turning the switch $A$ ') between two layers, except logical constraints and mapping functions provided as input.

\section{Problem Formulation}

\section{Combining C-space and State Space}

Here we suggest new problem formulation to combine Cspace of an object-manipulating robot and $\mathrm{KB}$ (defined in the next paragraph) of objects and propositions. An object, located in a specific workspace, generates propositions into KB. Other axioms (propositional formulas) and actions (PDDL(McDermott 1998)) are given for the propositions. We will call this KB as CPMP (Combining Planning and Motion Planning).

Definition CPMP (Combining Planning and Motion Planning) is composed of propositions for states of a robot and objects, logical axioms over a robot and objects, and PDDL actions of a robot. It groups a set of points in $\mathrm{C}$-space into a proposition $\left(p_{c}\right)$ in the $C P M P$. Actions of a robot are translated into actions of the CPMP. A set of propositions and actions are constrained each other by logical axioms (propositional formulas).

A CPMP is composed of propositional symbols, logical axioms, and PDDL actions. The propositional symbols $(P)$ represent states in binary values. The axioms (Axiom) are

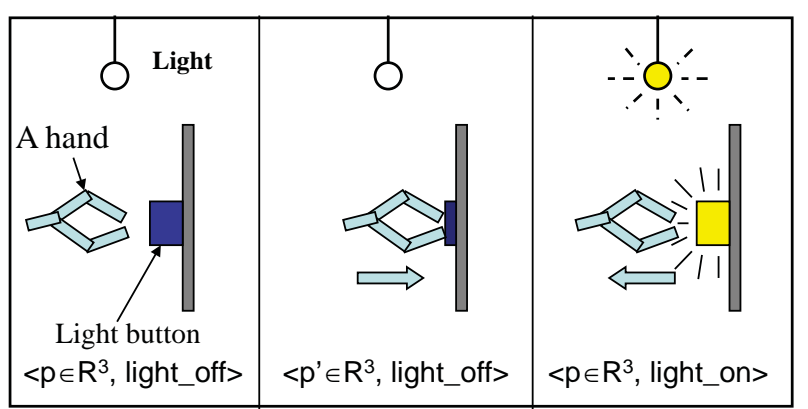

Figure 5: This example shows a situation in which one position in the workspace can correspond to two different states in the combined space (CPMP). Although the physical locations of the arm and button are the same in the workspace, the state (eg. light is on) is different. The situation can be represented when the $\mathrm{C}$-space and state space in $\mathrm{KB}$ are combined (CPMP), and it is not possible to represent in the classical $\mathrm{C}$-space alone.

propositional formulas. The actions (Action) represent the pair of preconditions and effects of a robot motion. It has a set of propositions that represents states of a robot and objects. External states are propositions in $K B_{M}$ extracted from $\mathrm{C}$-space. Internal states are propositions explicitly given in $K B_{O}$.

It also include a set of axioms. The axioms (logical formulas) represent relations among states of objects. When a state of an object $\left(o^{i}\right)$ is $o_{1}^{i}$ (e.g. light), the state of another object $\left(o^{j}\right)$ is constraints $o_{1}^{j}$ (e.g. $\neg$ shower). ${ }^{6}$ It is represented as follows.

$$
o_{1}^{i} \leftrightarrow o_{1}^{j}
$$

In CPMP, a set of actions, $K B_{M}$, is generated from a tree (or network) in C-space built by a motion planning algorithm as shown in figure 3 . In detail, two points ( $p_{1}$ and $p_{2}$ ) in the network are connected by a line (an action of the robot). This can be simply encoded as follows.

$$
\begin{array}{ll}
\text { Action }: & \text { Move }\left(p_{1}, p_{2}\right) \\
& \text { Precond }: p_{1} \\
& \text { Effect }: p_{2} \wedge \neg p_{1}
\end{array}
$$

When the action changes the state of an object $(o)$ from $o_{1}$ to $o_{2}$, the action can be encoded as follows.

$$
\begin{array}{ll}
\text { Action : } & \text { MoveObject }\left(p_{1}, p_{2}, o_{1}, o_{2}\right) \\
& \text { Precond }: p_{1} \wedge o_{1} \\
& \text { Effect }: p_{2} \wedge o_{2} \wedge \neg p_{1} \wedge \neg o_{1}
\end{array}
$$

Figure 5 represents the expressive power of $C P M P$. It represents a situation which can not be described by a $\mathrm{C}$ space but $C P M P$. The same physical locations are different states in $C P M P$ because the state of the light is changed.

A $C P M P$ has following properties.

\footnotetext{
${ }^{6}$ Such axioms are manually encoded. However, the encodings are independent of a specific robot. Thus, the encodings can be reusable to other types of robot. Moreover, there are algorithms (Amir and Russell 2003; Shahaf and Amir 2007) which can generate such axioms with a sensor-mounted robot.
} 


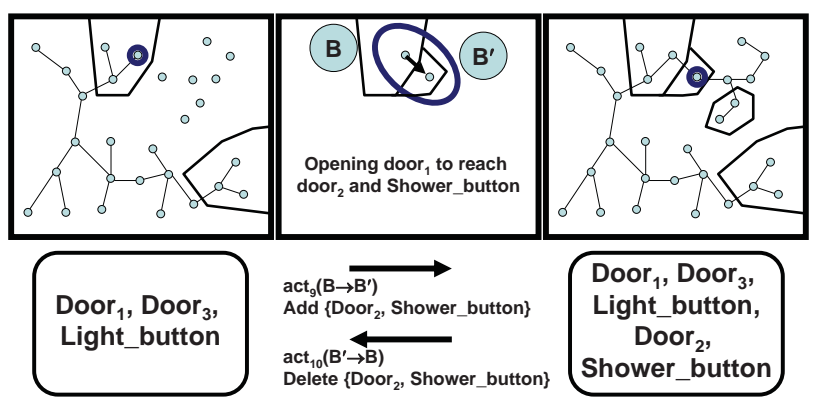

Figure 6: This figure represents an action which changes states of the object $\left(\right.$ Door $\left._{1}\right)$ to change the reachable set of objects. Before doing the action $\left(a c t_{9} B \rightarrow B^{\prime}\right)$, the set of reachable objects are \{ Door $_{1}$, Door $_{3}$, and Light_button $\}$. After the action, $\left\{\right.$ Door $_{2}$, Shower_button $\}$ are also included in the reachable set.

- A $C P M P$ has more expressive power than a C-space, if no two configurations in $\mathrm{C}$-space can distinguish the two internal states. $^{7}$

- It may reduce the number of propositions in $C P M P$, if spatial locations of end-effector are well-defined into disjoint sets. In each disjoint set, all spatial locations of endeffector have an identical internal state. Thus, any edge between the two disjoint sets changes some of the internal state.

Lemma 1. The complexity of planning problem in the $C P M P$ is as hard as P-SPACE.

Proof. Any motion planning problem (P-SPASE hard) with movable objects can be reduced to a planning problem in $C P M P$. Suppose that $C P M P$ includes only external propositions which are extracted from the motion planning algorithm.

\section{Encoding with Mapping Functions and Reachability}

Here, we suggest an automatic encoding for moving objects while maintaining states given mapping functions ${ }^{8}$ and reachability of objects. When a robot manipulates movable objects, it changes C-space of the robot. Hybrid systems(Alami, Siméon, and Laumond 1989; Alami, Laumond, and Siméon 1997; Alami et al. 1998) consider each C-space as a mode. Then, each manipulation connects two distinct modes. However, the size of the space is exponentially proportional to the number of objects and the number of joints. To address this issue, we group a set of modes based on the states of propositions and reachability of objects as shown in figure 3 and 6.

There are two cases to register an action (an edge between two points extracted from a motion planner) into CPMP. Firstly, we register an action into CPMP, if two points have different states in CPMP with a mapping function as shown

\footnotetext{
${ }^{7} \mathrm{C}$-space normally takes into account configurations which only consider spartial locations of a robot or objects.

${ }^{8} \mathrm{~A}$ mapping function provides a state of a proposition (eg. object) given a configuration of objects and a robot.
}

in figure 3. We validate abstract PDDL actions which are realized by the action. Secondly, we also register an action into CPMP, if an edge changes a set of reachable objects as shown in figure 6. ${ }^{9}$ Thus, we build a hypergraph whose nodes are sets of modes (C-spaces) which have the same states (in terms of mapping functions) and the same set of reachable object. Our algorithm extensively searches actions with a resolution complete motion planner until no new action is found in the hypergraph given a specific resolution.

Lemma 2. The size of the discretized C-space for a robot manipulating $n$ objects with given propositions in CPMP is bounded by $O(\exp (\mid$ objects $\mid+n+p))$, when $\mid$ objects $\mid$ is the number of objects, $n$ is the DOF (Degree of Freedom) of the robot, and $p$ is the number of propositions.

Lemma 3. The number of possible actions (edges) in the discretized C-space for objects is only bounded by $O((\mid$ objects $\mid) \cdot \exp (\mid$ objects $\mid))$, when the robot moves one object with an action.

Proof. From a point in C-space of object $O(\exp (\mid$ objects $\mid))$, we can choose an object $O(\mid$ objects $\mid)$ to change states.

\section{Finding a Solution in $C P M P$}

To solve a task in $C P M P$, we provide a naive algorithm followed by two improvements: (1) it solves the problem in the (smaller) factored KBs; and (2) it reduces the number of propositions in $C P M P$ using workspace.

\section{A Naive Solution}

Given a CPMP, algorithm NaiveSolution finds a solution for a task. It may use a general purpose planner (GeneralPlanner) to find an abstract solution. Then, (LocalMotionPlan) encodes a path in C-space.

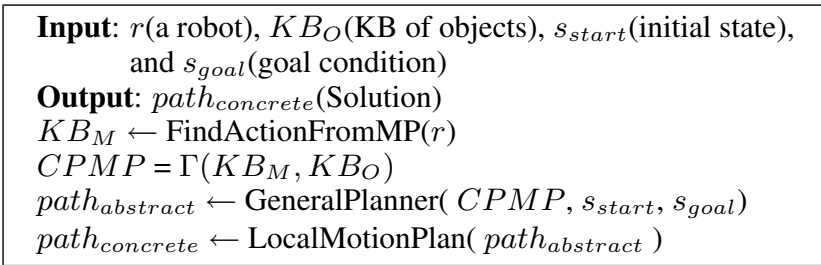

Algorithm 1: NaiveSolution provides a path for a robot. It uses a general planner (GeneralPlanner) to find an abstract solution. Then, it is encoded into the path in the $\mathrm{C}$-space by a motion plan (LocalMotionPlan).

\section{Tree Decomposition of KB with Objects}

Given a KB, finding a tree-decomposition of the minimum treewidth is a NP-hard problem. However, the complexity is only bounded by the treewidth of CPMP, if a treedecomposition is found once by an efficient heuristic(Becker and Geiger 1996; Amir 2001).

\footnotetext{
${ }^{9}$ The reachable objects are added to preconditions and effects respectively.
} 


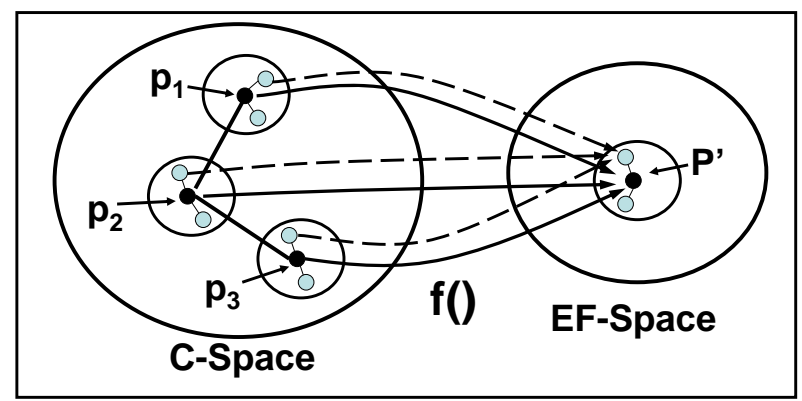

Figure 7: This figure shows a mapping function (f()) from a Cspace to an EF-Space. $p_{1}, p_{2}$, and $p_{3}$ in C-space are mapped into $p^{\prime}$ in EF-Space. The connected lines $\left(\left(p_{1}, p_{2}\right)\right.$ and $\left.\left(p_{2}, p_{3}\right)\right)$ represent the first condition of Theorem 3 . The circles represent the second condition.

Theorem 4. The complexity of planning in CPMP is bounded by $O(\exp (t w(C P M P)))$ if the treedecomposition is given. ${ }^{10}$

Proof. Proofs in (Brafman and Domshlak 2006; Amir 2001) can be easily modified to prove this theorem.

\section{From Exponential C-space to Polynomial EF-Space}

In this section, we provide an improvement for a previous approach(Choi and Amir 2007) which uses workspace instead of exponential C-space. Although it is not always applicable, it efficiently finds a solution when applicable. Here, we want to transform C-space into a smaller space, EF-Space, using a mapping function $f()$. The function $(f())$ maps each point $(p)$ in C-space into a point $\left(p^{\prime}\right)$ in EF-Space with satisfying following conditions.

1. Suppose that $P$ is a set of points whose image are $p^{\prime}$ in EF-Space $\left(f(p)=p^{\prime}\right)$. Any pair of two elements $\left(p_{1}, p_{2}\right.$ $\in P$ ) is connected each other in C-space;

2. Suppose that two points ( $p$ and $q$ ) are mapped into two points $\left(p^{\prime}\right.$ and $\left.q^{\prime}\right)$ in EF-Space. $p$ and $q$ are connected neighbor if and only if $p^{\prime}$ and $q^{\prime}$ are connected neighbor.

The connected neighbor means that they are directly connected in the space.

Theorem 5. The complexity of motion planning in EFSpace is bounded by following

$$
O(E F-S p a c e) \cdot O\left(\max _{e p \in E F-S p a c e}\left(\operatorname{ball}\left(P_{e p}\right)\right)\right) .
$$

$P_{e p}$ is a set of points whose image is ep. (That is, $P_{e p}=$ $\{p \mid f(p)=e p\})$ The ball $(P)$ is volume of the ball which includes $P$.

Proof. Given a motion planning problem (an initial configuration and goal one), a path in EF-Space can be found in $\mathrm{O}(\mathrm{EF}-\mathrm{Space})$ with a graph search algorithm. Given the path in EF-Space, one needs to search the whole ball in worst case.

One simple example of EF-Space is the workspace of endeffector. Suppose that the points in C-space are mapped

\footnotetext{
${ }^{10} \mathrm{tw}(\mathrm{KB})$ is the treewidth of $\mathrm{KB}$.
}

into the points of end-effector in workspace. One can build an algorithm that finds all the neighboring points from the innermost joint (or wheel) to the outermost joint with dynamic programming. If points of the previous joint are connected to all the neighboring points, the neighboring points of the current joint are found by a movement of current joint (current step) or a movement of any previous joint (previous steps). The found connected points in workspace satisfy the second conditions, if the first condition holds in the workspace.

In worst case, the first condition is hard to satisfy. In the environment, the mapping function $(f)$ should be bijective. Thus, the EF-Space is nothing but the C-space. However, the first condition holds in many environment where the distance between the obstacles (or object) and the robot is far enough. That is the theoretical reason why the planning problem in the spare environment is easy even in C-space.

Moreover, one can find another EF-Space considering topological shape of robot (Choi and Amir 2007). In the space, two points $\left(p_{1}\right.$ and $\left.p_{2}\right)$ are mapped into the same point $p_{1}^{\prime}$ if two configurations $\left(p_{1}\right.$ and $\left.p_{2}\right)$ are homotopic, and they indicate the same end point. Otherwise, another point $p_{2}^{\prime}$ is generated in the EF-Space. In $2 \mathrm{D}$, two groups of configurations are divided by an island in right and left slides. Thus, the EF-Space is exponential to the number of island obstacles. However, the EF-Space itself is bounded by the workspace which is polynomial to the number of joints. Thus, it is much smaller than the $\mathrm{C}$-space.

\section{A Unified Motion Plan}

We present our algorithms in this section. The main algorithm , UnifiedMotionPlanner (Algorithm 2), is composed of three parts: FindActionFromMP (Algorithm 3); FactoredPlan (Algorithm 4); and LocalPlanner. The goal of UnifiedMotionPlanner is to find a solution to achieve a goal situation.

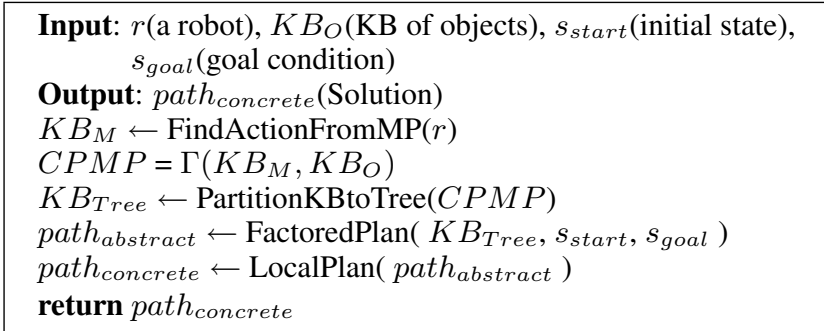

Algorithm 2: UnifiedMotionPlaner finds all the reachable locations and actions in each location with FindActionFromMP. A motion planner is embedded in FindActionFromMP to extract abstracted actions in $\mathrm{C}$-space. Then, PartitionKBtoTree partitions the $C P M P$ into a tree. FactoredPlan finds a solution given the pair of initial and goal condition in the partitioned tree domain. The LocalPlan finds a concrete path for the robot.

\section{FindActoinFromMP}

FindActionFromMP searches all the reachable locations and actions in C-space or EF-Space. In both cases, it has a dra- 


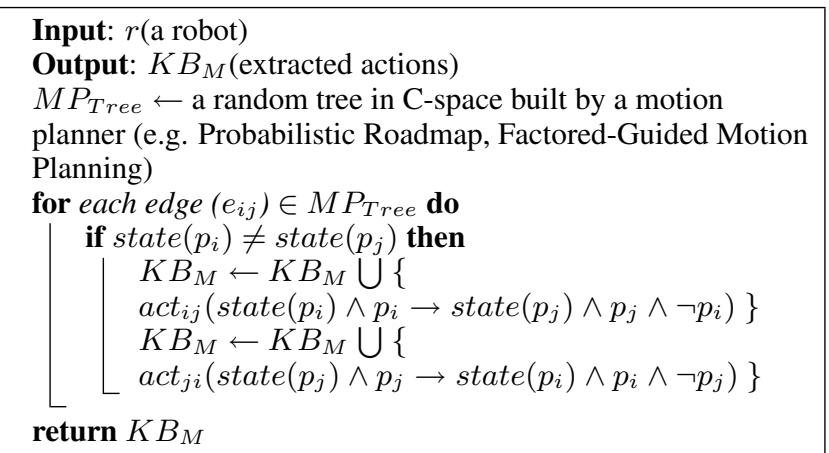

Algorithm 3: . FindActionFromMP finds all abstract actions for a robot. A motion planner (eg. FactorGuidedPlan or RoadmapMethod) recursively finds all the reachable locations and actions. Then, the algorithm insert actions of each configuration $\left(c_{i j}\right)$ of objects in the workspace. It assume that the object is in the configuration $\left(c_{i j}\right)$. Thus, the condition (configuration of objects) is combined into the actions $\left(a c t_{i j}\right)$. The union of all actions become the $K B_{M}$.

matically reduced space.

\section{FactoredPlan}

FactoredPlan finds a solution after factoring the domain (the space of end-effector in workspace) into small domains. It decomposes the domain into a tree in which each partitioned group becomes nodes, and shared axioms appear on a link between nodes. Then, it finds partial plans for a node and its children nodes with assuming that the parents nodes may change any shared states in between. After all, it finds a global solution in the root node.

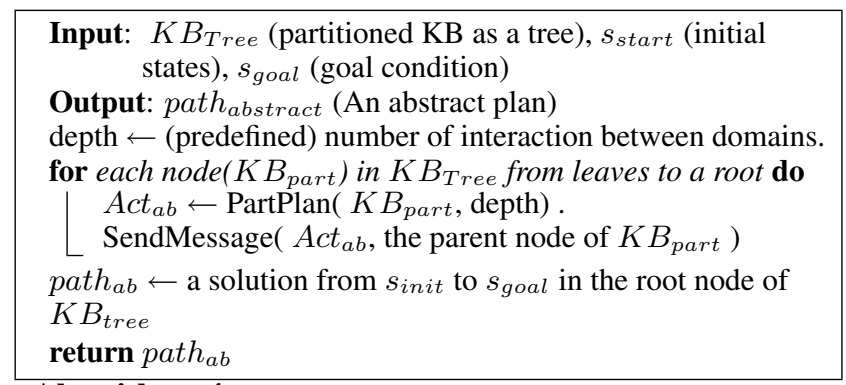

Algorithm 4: FactoredPlanning algorithm automatically partitions the domain to solve the planning problem (from $s_{\text {init }}$ to $s_{\text {goal }}$ ). It iterates domains from leaves to the root node without backtracks. In each node, PartPlan finds all possible actions that change shared states in the parents node. PartPlan assumes that the parent node may change any states in the shared states in between. The planned actions in the subdomain become an abstract action in the parent node. They are passed by SendMessage.

\section{An Experiment in Simulation}

In this preliminary simulation, we build our algorithm for a task that pushes buttons to call numbers. There are 8 buttons in total. 4 buttons $(k e y 1(P 1), \operatorname{key} 2(P 2)$, unlock $(P 3)$, and

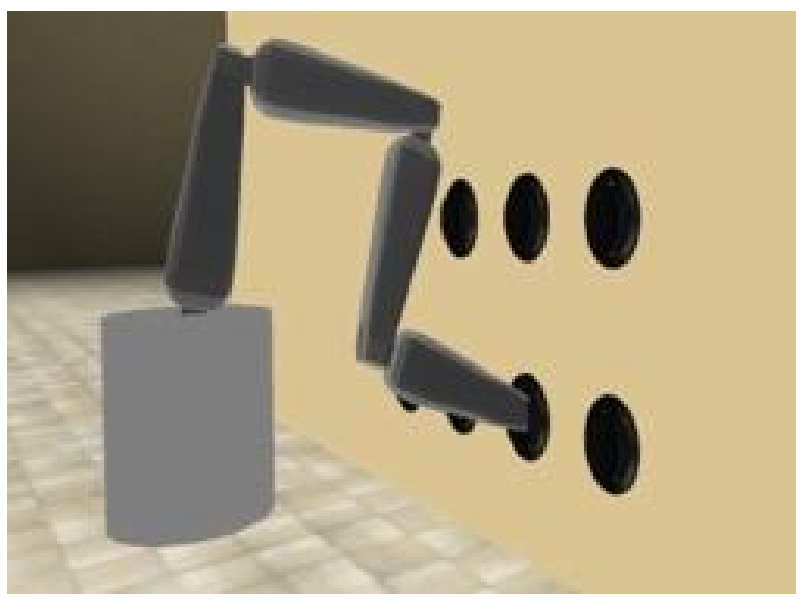

Figure 8: This is a capture of the motion of push button in the wall in experiments. The robot has 5 DOFs (rotational joints on the base and 4 revolute joints on the arm). We do experiment with increasing the number of joints from 2 to 9 .

lock $(P 4))$ are used to lock (and unlock) the buttons. Other 4 buttons (\#A(P5), \#B(P6), \#C(P7) and $C a l l(P 8))$ are used to make phone calls. Initially, the button is locked, the robot needs to push unlock buttons after pushing both key buttons $(P 1$ and $P 2)$. Then, the robot can make a phone call with pushing the $C$ all button $(P 8)$ after selecting an appropriate number among \#A(P5), \#B(P6), and \#C(P7). After a call, the buttons are automatically unlocked. We encode such constraints and action in $K B_{O}$.

To build $K B_{M}$, we build a tree from a randomized algorithm with 80000 points in C-space. With a labeling function that returned the states of buttons, we found 33 edges in the tree $^{11}$. They are encoded into 8 actions in $K B_{M}$ for 8 buttons. Then, the combined $\mathrm{KB}(C P M P)$ is used to find a goal (calling all numbers $(\# A, \# B$, and $\# C$ ). The returned abstract actions are decoded into a path on the tree of motion plan. Figure 8 is a snapshot of the simulation. ${ }^{12}$

In this experiment, we focus on extracting actions from a motion planning algorithm, because factored planer itself is not a contribution of this paper. Theoretical and experimental benefits of FactoredPlan is shown in the previous papers (Amir and Engelhardt 2003; Brafman and Domshlak 2006). We run our simulation on a general purposed planner (Fourman 2007). Thus, the NaiveSolution algorithm is used in this simulation.

\section{Conclusions and Future Research}

We present an algorithm that combines the general purpose (logical) planner and a motion planner. Our planner is designed to manipulate objects with robot. To solve the problem, previous works used a hierarchical planner (high-level) and a motion planner (low-level). Most of them used manual encodings between two layers. That was one of technical

\footnotetext{
${ }^{11}$ We simplify the manipulations for attaching and detaching buttons

${ }^{12}$ The details of encoded actions and movies are available at http://reason.cs.uiuc.edu/jaesik/cpmp/supplementary/.
} 
hardness of this problem.

Theoretically, the combination of such planner is hard for the following reasons: (1) hierarchical planner is hard and not feasible sometime; and (2) direct combination of $\mathrm{C}$-space and state space gives an doubly exponential search problem. Moreover, we can miss the geometric motion planning information, if we translate everything to PDDL (McDermott 1998) without a motion planner.

We combine the C-space and state space in a KB, CPMP (Combining Planning and Motion Planning). Moreover, we provide the computational complexity of the problem. We also argue that the treewidth of CPMP determines the hardness of a manipulation task.

The suggested algorithm still has some limitations that need to be improved in future research. First, mapping function in Section 4needs manual encodings. Our algorithms assume that there is a mapping function which provides the value of shared propositions given a configuration of C-Space. Thus, an algorithm which can detect the change of shared propositions with sensors would be promising. Second, the exploration steps in FindActionFromMP may take long time due to the large cardinality of state space $(O(n+\mid$ objects $\mid+p)$ as in lemma 2. Third, assumptions of EF-space would inappropriate for cluttered environments where $O\left(\max _{e p \in \mathrm{EF}-\mathrm{Space}}\left(\operatorname{ball}\left(P_{e p}\right)\right)\right.$ of theorem 5 are intractable.

The combining planning and motion planning is a generalized framework. However, there are many research problems to be solved in the future research. First, an algorithm which learns the mapping function between two spaces is necessary. Our algorithm assumes that there is a mapping function which provides the value of shared propositions given a configuration of C-Space. Thus, an algorithm which can detect the change of shared propositions with sensors would be promising. Second, the exploration step may take long time due to the large cardinality of state space. Thus, an adaptive exploration algorithm which builds a tree or a graph in CSpace based on the constraints of stats space would be useful.

\section{Acknowledgment}

This material is based upon work supported by the National Science Foundation under Grant No. 05-46663. We also thank UIUC/NCSA Adaptive Environmental Sensing and Information Systems (AESIS) initiative for funding part of the work.

\section{References}

Alami, R.; Chatila, R.; Fleury, S.; Ghallab, M.; and Ingrand, F. 1998. An architecture for autonomy. International Journal of Robotics Research 17(4):315-337.

Alami, R.; Laumond, J.-P.; and Siméon, T. 1997. Two manipulation planning algorithms. In Laumond, J.-P., and Overmars, M., eds., Algorithms for Robotic Motion and Manipulation. Wellesley, MA: A.K. Peters.

Alami, R.; Siméon, T.; and Laumond, J.-P. 1989. A geometrical approach to planning manipulation tasks. In Proceedings International Symposium on Robotics Research, 113-119.

Amir, E., and Engelhardt, B. 2003. Factored planning. In IJCAI, 929-935.
Amir, E., and Russell, S. J. 2003. Logical filtering. In IJCAI, $75-82$.

Amir, E. 2001. Efficient approximation for triangulation of minimum treewidth. In UAI, 7-15.

Becker, A., and Geiger, D. 1996. A sufficiently fast algorithm for finding close to optimal junction trees. In $U A I, 81-89$.

Brafman, R. I., and Domshlak, C. 2006. Factored planning: How, when, and when not. In $A A A I$.

Brock, O., and Khatib, O. 2000. Real-time replanning in highdimensional configuration spaces using sets of homotopic paths. In ICRA'00, 550-555.

Canny, J. 1987. The Complexity of Robot Motion Planning. Cambridge, MA: MIT Press.

Chen, P., and Hwang, Y. 1991. Motion planning for a robot and a movable object amidst polygonal obstacles. In ICRA'91, 444-449.

Choi, J., and Amir, E. 2007. Factor-guided motion planning for a robot arm. In IROS'07, 27-32.

Conner, D. C.; Kress-Gazit, H.; Choset, H.; Rizzi, A.; and Pappas, G. J. 2007. Valet parking without a valet. In IROS'07.

Cortés, J. 2003. Motion Planning Algorithms for General ClosedChain Mechanisms. Ph.D. Dissertation, Institut National Polytechnique de Toulouse, Toulouse, France.

Dacre-Wright, B.; Laumond, J.-P.; and Alami, R. 1992. Motion planning for a robot and a movable object amidst polygonal obstacles. In ICRA'92, volume 3, 2474-2480.

Dejong, G., and Mooney, R. 1986. Explanation-based learning: An alternative view. Mach. Learn. 1(2):145-176.

Fourman, M. 2007. Propplan. Software.

J. Van den Berg, M. O. 2007. Kinodynamic motion planning on roadmaps in dynamic environments. In IROS'07.

Kavraki, L. E.; Svestka, P.; Latombe, J.-C.; and Overmars, M. 1996. Probabilistic roadmaps for path planning in high dimensional configuration spaces. IEEE Trans. on Rob. and Auto. 12(4):566580 .

Kuffner, J. J., and LaValle, S. M. 2000. RRT-connect: An efficient approach to single-query path planning. In ICRA'00.

Likhachev, M.; Gordon, G.; and Thrun, S. 2003. ARA*: Anytime A* search with provable bounds on sub-optimality. In NIPS'03.

M. Pardowitz, R. Zollner, R. D. 2007. Incremental acquisition of task knowledge applying heuristic relevance estimation. In IROS'07.

McDermott, D. 1998. The planning domain definition language manual.

Plaku, E.; Kavraki, L. E.; and Vardi, M. Y. 2008. Hybrid systems: From verification to falsification by combining motion planning and discrete search. Formal Methods in System Design.

S. Hart, R. G. 2007. Natural task decomposition with intrinsic potential fields. In IROS'07.

Shahaf, D., and Amir, E. 2007. Logical circuit filtering. In IJCAI, 2611-2618.

Stilman, M., and Kuffner, J. 2005. Navigation among movable obstacles: Real-time reasoning in complex environments. International Journal of Humanoid Robotics 2(4):479-504.

Stilman, M. 2007. Task constrained motion planning in robot joint space. In IROS'O7. 